\title{
Evaluation of Labor Units of Competency: Facilitating Integration of Disabled People
}

\author{
Amparo Jiménez, Amparo Casado, Fernando de la Prieta, \\ Sara Rodríguez, Juan F. De Paz, and Javier Bajo
}

\begin{abstract}
Education and training for disabled people has acquired a growing relevance during the last decade, especially for labour integration. Disabled people represent a considerable percentage of the current population and require special education. This paper presents a competencies model to evaluate the skills of disabled people previous to their integration to working scenarios.
\end{abstract}

Keywords: units of competency, disabled, active learning.

\section{Introduction}

Remote training methods acquire an important role nowadays, as they allow the users to interact with the workplace before facing reality. Therefore, one of the objectives to be achieved is to obtain an active learning methodology via internet or TV proposing innovative learning strategies that enable people with visual, hearing and motor disabilities to perform training and learning prior to their employment. This paper proposes a novel learning method that combines the advantages of active learning with the technological capabilities of adapted devices. Active learning allows the users to learn for themselves and acquire knowledge through their own experiences. In addition, the introduction of interactive channels is made available to all the users as a mechanism for communication and interaction with great audio-visual possibilities.

Amparo Jiménez · Amparo Casado · Javier Bajo

Universidad Pontificia de Salamanca, Salamanca, Spain

e-mail: $\{a j$ imenezvi, acasadome, jbajope $\}$ ausal.es

Fernando de la Prieta · Sara Rodríguez · Juan F. De Paz

Departamento Informática y Automática,

Universidad de Salamanca, Salamanca, Spain

e-mail: $\{$ fer, srg, fcofds, corchado\}@usal.es

J.B. Pérez et al. (Eds.): Highlights on PAAMS, AISC 156, pp. 281-288.

springerlink.com @ Springer-Verlag Berlin Heidelberg 2012 
Without going into specific definitions of what skills are or what their different levels of specification, evaluation and training, an essential step in the career counseling of people with disabilities, is to clearly determine the job profile to develop. Depending on the definition of the job position and the desired profile, it is possible to propose a model for assessing competence. It is necessary to consider, very specifically, which are the functions of the worker and, from that description, provide the technological resources needed to carry out the work.

The aim of this paper is to propose a teaching method for designing a new panel of execution of exercises based on active learning methodologies that can adapt to the characteristics of the disabled user that runs them. The approach will provide a new technological solution for training and distance learning, and opens a wide range of research into new methods of training disabled people. We propose an adaptive mechanism incorporating new algorithms at each stage of a case-based reasoning. We propose the use of cooperative Hebbian learning [12] in the recovery phase and SOM neural networks [11] incorporating new modifications for reuse phase.

The rest of the paper is organized as follows: Section 2 revises the related work. Section 3 describes the proposed approach. Finally, section 4 presents the initial conclusions obtained.

\section{Related Work}

There is an ever growing need to supply constant care and support to the disabled and the drive to find more effective ways to provide such care has become a major challenge for the scientific community [5]. Education is the cornerstone of any society and it is the base of most of the values and characteristics of that society. The new knowledge society offers significant opportunities for AmI applications, especially in the fields of education and learning [7]. The new communication technologies propose a new paradigm focused on integrating learning techniques based on active learning (learning by doing things, exchange of information with other users and the sharing of resources), with techniques based on passive learning (learning by seeing and hearing, Montessori, etc.) [6]. While the traditional paradigm, based on a model focused on face to face education, sets as fundamental teaching method the role of the teachers and their knowledge, the paradigm based on a learning model highlights the role of the students. In this second paradigm the students play an active role, and build, according to a personalized action plan, their own knowledge. Moreover, they can establish their own work rhythm and style. The active methodology proposes learning with all senses (sight, hearing, touch, smell and taste), learn through all possible methods (school, networking, etc.), and have access to knowledge without space or time restrictions (anywhere and at any time).

More and more learning platforms are designed for use by disabled people, given the ease of using and the increase of technology in our daily lives. Moreover, in the case of young children, an electronic device as a tablet or smartphone as a learning tool can be especially appropriated, given the attraction of these devices. Currently there are several types of platforms for education for 
the disabled. There are games based platforms as PICAA [4]. It is an application for smartphones (iPhone or Apple iPod Touch). Other platforms are based on exercises like PROAF (Program Articulation and Phonation) [2]. This program is conducted from the Office suite that offers Microsoft, PowerPoint tool, which allows us to create presentations. The program P.R.O.A.F. is for students with poor auditory skills and language disorders [2]. In 2009, a student of the UNEFMaracay, developed by the end of grade one braille learning platform for the blind and deaf blind [3]. The O.N.C.E. (National Organization of Spanish Blind) makes available to its members a range of applications for global development, employment, education and social development. These resources are open to anyone who wants to use them [1]. Among them, we can distinguish from braille printer drivers to games and stories for children through voice synthesizer applications.

\section{Proposed Mechanism Overview}

Without going into specific definitions of what skills are or what their different levels of specification, evaluation and training, an essential step in the career counseling of people with disabilities, is to clearly determine the job profile to develop. Depending on the definition of the job position and the desired profile, it is possible to propose a model for assessing competence. It is necessary to consider, very specifically, which are the functions of the worker and, from that description, provide the technological resources needed to carry out the work.

To this end, an essential step in this description is to place ourselves in a particular model of time and labor needs. In Spain, as in other countries, there exists a framework document that determines the qualifications of individual workers, and that unifies and determines what "to do" in the various work environments. Therefore, we understand that when addressing the issue of training for employment, we must take as reference the proposal presented in the Spanish document of INCUAL (National Institute of Professional Qualifications) [14]. This document allows us to indicate the structure of the professional qualifications that will be used to design the programs, resources, methodologies and educational interventions in labor scenarios. Every qualification is associated with a general competence, which briefly defines the duties and essential functions of the worker. We also need to describe the professional environment in which the workers can develop their skills, the corresponding productive sectors and occupations or relevant job positions to which they will be able to gain access. Each qualification is divided into units of competency. The unit of competency is the minimum aggregate of professional skills, capable of being recognized, being partially accredited and, in training environments, to training and assessment. Each unit of competency has associated a training design (no training module as in the SNCP), which describes the necessary training to acquire this unit of competency. In this case, each unit of competency will be associated with different technology-based educational resources that allow individuals to learn or verify their appropriateness in the performance taking into account the special characteristics of the target persons, who have disabilities in this case. The unit of 
competency is divided into professional achievements. The personal achievements describe the expected behaviors of the person, objectified by its consequences or outcomes, so that it can be considered competent in that unit.

Thus, we propose as valid a proposal following the guidelines of the Spanish National Catalogue of Professional Qualifications (CNCP), since it is the instrument used by the Spanish National System of Qualifications and Vocational Training (SNCFP) that regulates the professional qualifications that can be recognized and accredited, identified in the production system based on appropriate competencies for professional practice. Taking this proposal as starting point, the next step is to determine, from the units of competencies described, the professional achievements and performance criteria selected from those identified in the CNCP. This selection allows us to determine which strategies and training resources any company can include in the training and development plans for their workers, or for the selection of new candidates. We must emphasize the current interest in technology-based design tools (current, new and technically valid) to help people with certain disabilities to effectively integrate in labor environments and job profiles.

\subsection{A Specific Competencies Model}

We have selected a professional qualification: Auxiliary operations and general administrative services, associated with the following occupations or relevant jobs: Office Assistant, General Services Assistant, Archives Assistant, Classifier and / or delivery of mail, Ordinance, Information Assistant, Telephonist, Box office. Within this first professional qualification the following units of competence are identified, which are associated to a certain performance criteria:

-UC0969_1: Conduct and integrate basic administrative support operations

- UC0970_1: Transmit and receive routine operational information with external efforts of the organization

- UC0971_1: Perform additional reproduction and archiving on conventional or compute

By means of an initial analysis work we select the most relevant professional achievements related to the particular disability. We identify as relevant those requiring greater effort in their level of training, learning and achievement in relation to the particular characteristics of the professionals who must perform these tasks. These are:

RP 1: Register periodically updates on information of the organization, its departments and areas, and staff assigned to them, as instructed, to have the information necessary to provide good service.

RP 2: Collate and record, business, administrative, financial, or other documents in the organization, as instructed, using specific or generic software, to ensure the reliability of the information. 
RP 3: Draw up notices, alerts, informational signs or other internal documents used in the communication process, following the instructions and using conventional means or computers.

RP 4: Maintain optimum operating conditions of equipment and multimedia resources, detecting and correcting incidents or managing repairs, according to the instructions received, in order to support the normal functioning of the office.

RP 5: Archiving and accessing both traditional and digital documentation, introducing specific codes, and following the procedures of the organization and the instructions received, so as to allow a quick search.

RP 6: Archivar y acceder a la documentación, en soporte convencional o digital, introduciendo los códigos precisos y respetando los procedimientos de la organización y las instrucciones recibidas, de manera que se permita una búsqueda rápida.

PR 7: Transfer and purge of documents according to the rules and deadlines and instructions, to maintain updated and functional the file system.

RP 8: Update and extract different types of information, using computer applications or databases, according to the rules and instructions, to display and query the information stored, and to facilitate upon request.

RP 9: Get the necessary copies of working documents on conventional or computational supports, in the quantity and quality required by the characteristics of the document itself, and according to the instructions given in accordance with applicable environmental protection.

RP 10: Scan or digitally reproducing documents, ensuring the highest quality of service according to the manuals and received instructions, in order to preserve and archive.

\subsection{A Reasoning Mechanism}

Case-based Reasoning is a type of reasoning based on the use of past experiences [9]. The fundamental concept when working with case-based reasoning is the concept of case. A case can be defined as a past experience, and is composed of three elements: A problem description which describes the initial problem, a solution which provides the sequence of actions carried out in order to solve the problem, and the final state which describes the state achieved once the solution was applied. The way in which cases are managed is known as the case-based reasoning cycle. This CBR cycle consists of four sequential steps: retrieve, reuse, revise and retain. The retrieve phase starts when a new problem description is received. Similarity algorithms are applied in order to retrieve from the case's memory the cases with a problem description more similar to the current one. Once the most similar cases have been retrieved, in the reuse phase the solutions of the cases retrieved are adapted to obtain the best solution for the current case. The revise phase consists of an expert revision of the solution proposed. Finally, 
the retain phase allows the system to learn from the experiences obtained in the previous phases and updates the case memory in consequence.

We propose an adaptive mechanism that incorporates new algorithms at each stage of a case-based reasoning system. We propose the use of cooperative Hebbian learning [12] in the recovery stage and SOM neural networks [10, 11] incorporating new modifications in the reuse stage, and CART [13] for knowledge extraction. Thus, we propose an active learning method for disabled people, proposing an adaptable learning model with great customization capabilities. The proposed mechanism uses the user profile and the services available and requested, it is possible to provide the best learning plan to facilitate their labor integration. The mechanism is mainly composed of a case-based reasoning system, which can be integrated into CBR-BDI agents [8], specifically designed to provide advanced reasoning mechanisms.

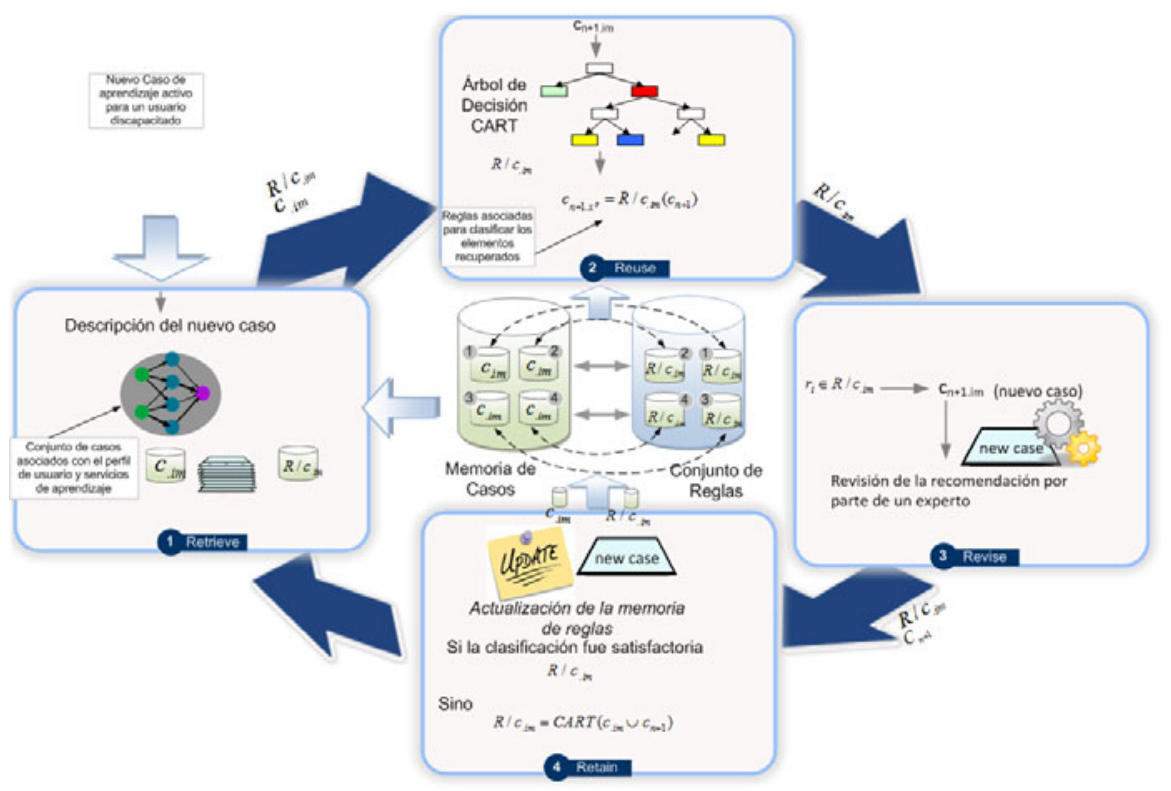

Fig. 1 Proposed mechanism to recommend training tasks.

A case is composed of the following elements: User profile (based on dissabilities, etc.), Available resources, Units of Competency, Professional achievements. The problem description consists of the user profile and units of competency taken into account in the workplace. Thus, a new problem seeks to find the solution that best suits the user's and work environment characteristics (described by the units of competency to be taken into account). The professional achievements are also taken into consideration. The solution consists of the most appropriate performance criteria for the user and the tools that were more appropriated for the user. The solution describes the professional achievements 
more appropriated for the user and the tools considered most suitable for carrying out the professional activities. The efficiency of the solution evaluates the degree of satisfaction and is provided by an expert. Thus, a case is defined as:

Table 1 Case Description

\begin{tabular}{llc}
\hline Fields & Type & Variable \\
\hline \hline IDCase & Int & $i$ \\
Profile_Type & String & $m$ \\
Disability_Type & Int & $s$ \\
Units_of_Competency & Array of Boolean & $n$ \\
Professional_Achievements & Array of Boolean & $l$ \\
Tools & Array of Boolean & $u$ \\
Achievement_Criteria & Array of Integer & $c$ \\
Efficiency & Double & $k$ \\
\hline
\end{tabular}

\section{Conclusions}

This paper has proposed a teaching method for designing a new panel of execution of exercises based on active learning methodologies that can adapt to the characteristics of the disabled user that runs them. The approach provides a new technological solution to support training and distance learning of disabled people. We have revised the related work and it was not possible to find existing approaches solving the problem of providing a generic solution for training of disabled people. In this paper we have focused on analyzing the units of competency and personal achievements for a specific professional qualification. The proposed model has been used to design a technological solution, that makes use of a case-based reasoning system, Hebbian learning, SOM artificial neural networks and CART to provide recommendations about panels of exercises taking into account previous experiences. We propose an adaptive mechanism incorporating new algorithms at each stage of a case-based reasoning.

Our future work focuses on evaluating the proposed approach in a case study scenario. This will help to have a quantitative evaluation of the proposal. Moreover, we will focus on tuning and improving the proposed model, as well as its possible extension to additional professional qualifications.

Acknowledgements. This project has been supported by the Spanish CDTI. Proyecto de Cooperación Interempresas. IDI-20110343, IDI-20110344, IDI-20110345. Project supported by FEDER funds.

\section{References}

1. Once (2011), ftp: / / ftp. once.es/pub/utt/tiflosoftware/

2. Proaf (2011), http://www. educarm.es/templates/portal/images/ ficheros/diversidad/10/secciones/11/contenidos/625/proaf .pps 
3. Rondon, R.: (2009),

http: / tecnologiasdeapoyodiscapacidad.blogspot.com/

2009/05/plataforma-electronica-de-aprendizaje.html

4. Rodríguez-Fórtiz, M.J., Fernández-López, A., Rodríguez, M.L.: Mobile Communication and Learning Applications for Autistic People. In: Williams, T. (ed.) Autism Spectrum Disorders - From Genes to Environment. InTech (2011) ISBN: 978953-307-558-7

5. Bajo, J., Molina, J.M., Corchado, J.M.: Ubiquitous computing for mobile environments. In: Issues in Multi-Agent Systems: The AgentCities. ES Experience. Whitestein Series in Software Agent Technologies and Autonomic Computing, pp. 33-58. Birkhäuser, Basel (2007)

6. Brown, T.H.: Beyond constructivism: Exploring future learning paradigms. Education Today 2005(2) (2005)

7. Friedewald, M., Da Costa, O.: Science and Technology Roadmapping: Ambient Intelligence in Everyday Life (AmI@Life). In: Working Paper. Institute for Prospective Technology Studies IPTS, Seville (2003)

8. Laza, R., Pavón, R., Corchado, J.M.: A Reasoning Model for CBR_BDI Agents Using an Adaptable Fuzzy Inference System. In: Conejo, R., Urretavizcaya, M., Pérez-de-laCruz, J.-L. (eds.) CAEPIA/TTIA 2003. LNCS (LNAI), vol. 3040, pp. 96-106. Springer, Heidelberg (2004)

9. Kolodner, J.: Case-Based Reasoning. Morgan Kaufmann, San Francisco (1983)

10. Kohonen, T.: Self-Organization and Associative Memory. Springer, Berlin (1984)

11. Baruque, B., Corchado, E.: A weighted voting summarization of SOM ensembles. Data Mining and Knowledge Discovery 21(3), 398-426 (2010)

12. Corchado, E., Baruque, B., Yin, H.: Boosting Unsupervised Competitive Learning Ensembles. In: de Sá, J.M., Alexandre, L.A., Duch, W., Mandic, D.P. (eds.) ICANN 2007, Part I. LNCS, vol. 4668, pp. 339-348. Springer, Heidelberg (2007)

13. Breiman, L., Friedman, J., Olshen, A., Stone, C.: Classification and regression trees. Wadsworth International Group, Belmont (1984)

14. INCUAL (National Institute of Professional Qualifications), https: / /www.educacion.es/iceextranet/ accesoExtranetAction.do 